\title{
Noncontact Free-Rotating Disk Triboelectric Nanogenerator as a Sustainable Energy Harvester and Self-Powered Mechanical Sensor
}

\author{
Long Lin, ${ }^{\dagger, \S}$ Sihong Wang, ${ }^{\dagger, \S}$ Simiao Niu, ${ }^{\dagger, \S}$ Chang Liu, ${ }^{\dagger}$ Yannan Xie, ${ }^{\dagger}$ and Zhong Lin Wang ${ }^{*}, \dagger$, \\ ${ }^{\dagger}$ School of Materials Science and Engineering, Georgia Institute of Technology, Atlanta, Georgia 30332-0245, United States \\ ${ }^{\ddagger}$ Beijing Institute of Nanoenergy and Nanosystems, Chinese Academy of Sciences, Beijing 100083, China
}

Supporting Information

ABSTRACT: In this work, we introduced an innovative noncontact, free-rotating disk triboelectric nanogenerator (FRD-TENG) for sustainably scavenging the mechanical energy from rotary motions. Its working principle was clarified through numerical calculations of the relative-rotation-induced potential difference, which serves as the driving force for the electricity generation. The unique characteristic of the FRD-TENG enables its high output performance compared to its working at the contact mode, with an effective output power density of $1.22 \mathrm{~W} / \mathrm{m}^{2}$ for continuously driving 100 light-emitting diodes. Ultrahigh stability of the output and exceptional durability of the device structure were achieved, and the reliable output was utilized for fast/effective charging of a lithium ion battery. Based on the relationship between its output performance and the parameters of the mechanical stimuli, the
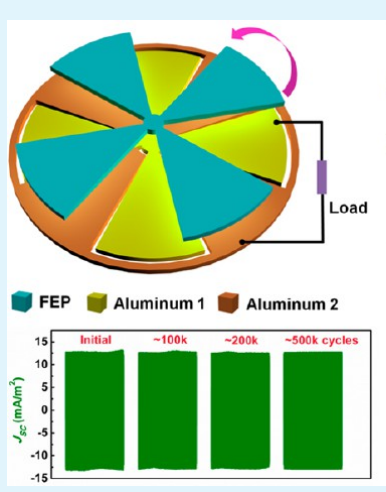
FRD-TENG could be employed as a self-powered mechanical sensor, for simultaneously detecting the vertical displacement and rotation speed. The FRD-TENG has superior advantages over the existing disk triboelectric nanogenerator, and exhibits significant progress toward practical applications of nanogenerators for both energy harvesting and self-powered sensor networks. KEYWORDS: triboelectric nanogenerator, noncontact, rotational mechanical energy, self-powered system, high stability, active sensor

\section{INTRODUCTION}

The worldwide energy crises and environmental pollution have drawn long-lasting attention to green energy and sustainable development. ${ }^{1,2}$ Harvesting energy from the ambient environment is a good choice to solve the energy-related problems without causing unexpected environmental issues. ${ }^{3-6}$ Among multiple energy sources, mechanical energies are universally available, environmental friendly, and can be scavenged through various approaches based on the piezoelectric, ${ }^{7-9}$ electrostatic, ${ }^{10,11}$ and electromagnetic effects. ${ }^{12-14}$ The recently invented triboelectric nanogenerator (TENG) $)^{15-18}$ has been proven to be an effective method for converting the mechanical agitations into electricity by the conjunction of contact electrification and electrostatic induction. ${ }^{19}$ However, most of the existing configurations of TENGs are designed for harvesting energy from an oscillating linear motion that realizes periodic separations of triboelectically charged (tribo-charged) surfaces in $\operatorname{contact}^{20}$ or sliding ${ }^{21}$ modes, while a rotary mechanical energy is also widely available in our daily life (like wheels, turbines, bearings, etc). Although we have demonstrated the segmentally structured disk triboelectric nanogenerator (D-TENG $)^{22}$ on the basis of the in-plane charge separation mechanism for such applications, its output performance, especially the stability/durability, still needs further improvement for long-term working. The limitations of such D-TENG exist in the following two aspects: (1) both the rotational and stationary triboelectric layers require deposition of metal electrodes and connection with electrical leads, leading to inconvenient operation of the rotational part; (2) intimate contact is mandatory to achieve efficient electricity generation, which results in possible material abrasion, instability of output, and limited lifetime of the TENG.

To resolve the above issues for the existing D-TENG, in this work we designed a novel type of disk TENG with both groups of patterned electrodes attached onto a stationary disk, together with a freestanding triboelectric layer on a rotational disk. With such a structure, there is no necessity for electrode deposition or electrical connection for the rotational part, which dramatically improves the operating facility of the energy harvester. Moreover, owing to the unique feature of this new electricity-generation mechanism, the noncontact free-rotating disk triboelectric nanogenerator (FRD-TENG) can be operated without friction after initial contact electrification, with little lost on performance but superior durability enhancement, because the surface triboelectric charges preserve on insulator surfaces for hours. ${ }^{23}$ Under the noncontact condition, an opencircuit voltage $\left(V_{\mathrm{OC}}\right)$ of $220 \mathrm{~V}$ and a short-circuit current density $\left(J_{\mathrm{SC}}\right)$ up to $13 \mathrm{~mA} / \mathrm{m}^{2}$ were produced, with an effective output power density of $1.22 \mathrm{~W} / \mathrm{m}^{2}$, which is enough for instantaneously and continuously driving 100 serially connected

Received: December 7, 2013

Accepted: January 20, 2014

Published: January 27, 2014 


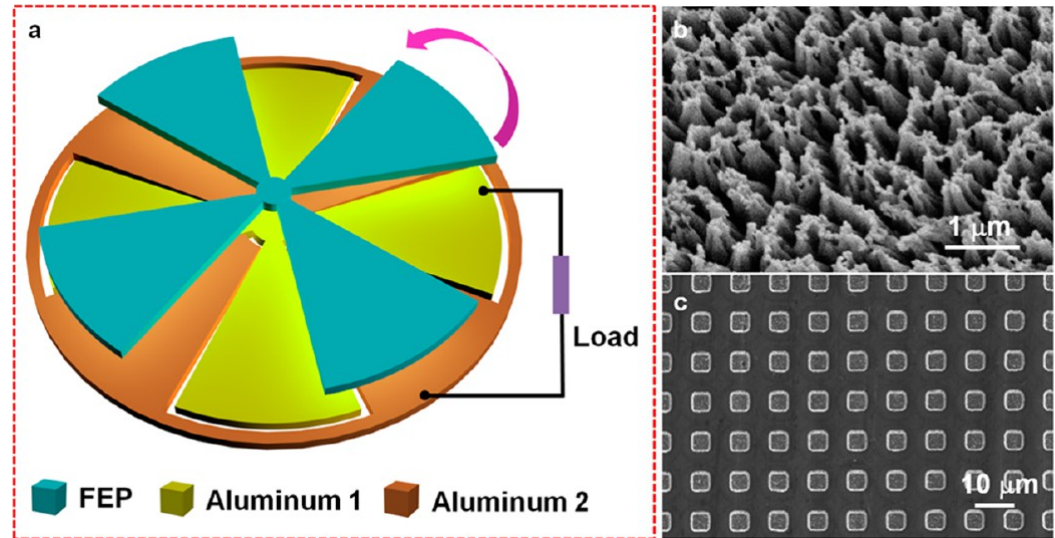

Figure 1. Device structure of the noncontact free-rotating disk triboelectric nanogenerator. (a) A schematic of the basic structure of the FRD-TENG composed of the freestanding FEP layer and the stationary aluminum layer. The bottom inset is the figure legend. (b) A $30^{\circ}$-tilted-view SEM image of the nanorod structure created on the surface of the FEP thin film. (c) A top view SEM image of the cubic micropatterned structures on the aluminum foil.
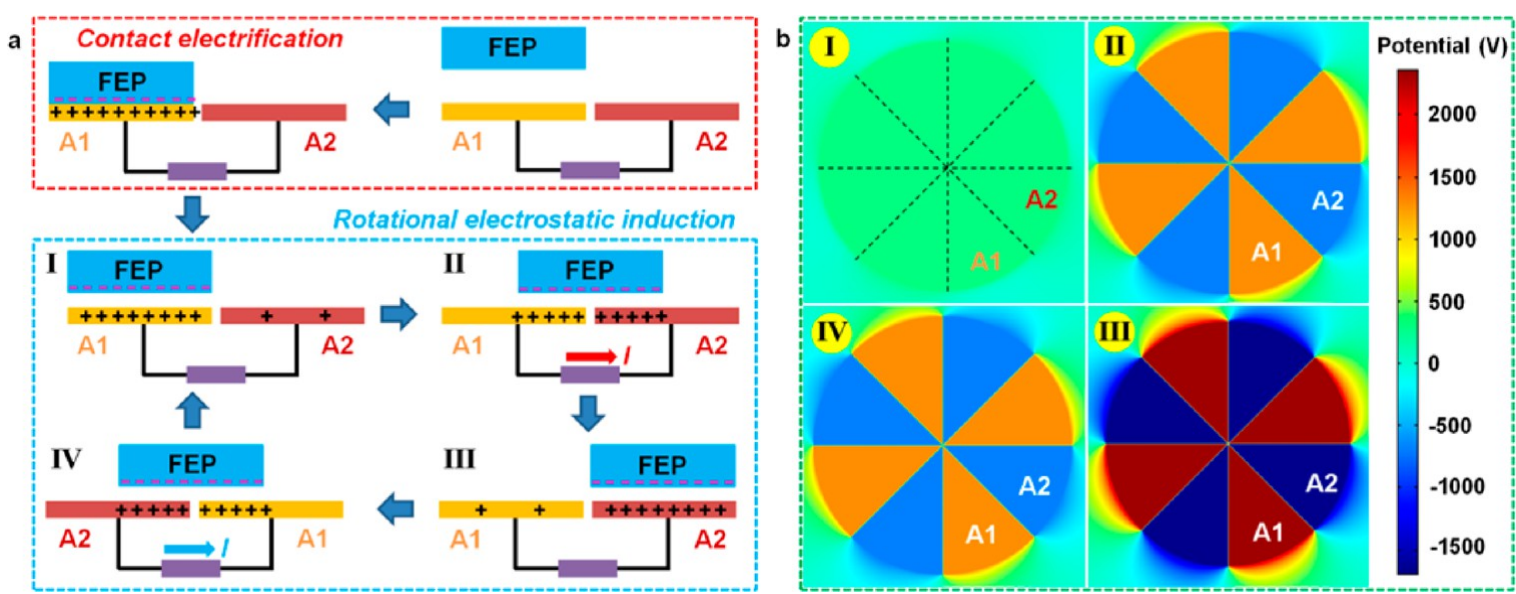

Figure 2. Theoretical study on the working principle of the FRD-TENG. (a) Schematic illustrations showing the working principle of the FRDTENG in a full cyclic motion of the rotational disk. (b) Numerical calculations on the induced potential differences between the two aluminum electrodes at the four steps of motion in a full cycle. The right inset shows the color legend about the value of potential.

light-emitting diodes (LEDs). The high output power remains stable upon continuous working for 500000 cycles and is capable of fully charging a lithium ion battery (LIB) within 1.5 $\mathrm{h}$, with an equivalent DC charging current of $7.5 \mu \mathrm{A}$. Finally, the FRD-TENG can be employed as a self-powered mechanical sensor for simultaneously detecting the vertical separation distance between the two disks and the rotation speed, through reading the magnitude and the frequency of the measured $J_{\mathrm{SC}}$. This work presents an outstanding progress toward the practical applications of nanogenerators in harvesting rotational mechanical energy, and boosts the development of selfpowered sensor networks, ${ }^{24-26}$ especially for application in the braking systems of automobile industry.

\section{RESULTS AND DISCUSSION}

As schematically illustrated in Figure 1a, the basic structure of the FRD-TENG consists of two parts: the freestanding rotational part of tribo-charged layer and the stationary part of metal electrodes. The rotational part was fabricated from a piece of fluorinated ethylene propylene (FEP) thin film $(\sim 50$ $\mu \mathrm{m}$ in thickness), which was tailored into a four-segment structure and attached onto an acrylic supporting substrate with same shape. In this new structure, there is no need to deposit electrode on the back surface of the FEP membrane, indicating that versatile energy harvesting is achievable with an arbitrary moving object without processing (e.g., rotating tires). The stationary part is composed of two separated aluminum foils $(\sim 50 \mu \mathrm{m}$ in thickness and 4 in. in diameter $)$ with complementary four-segment shapes attached on a roundshape acrylic disk, which act as the two stationary electrodes. To increase the density of triboelectric charges through enhanced electrification process, micropatterned structures were introduced on the inner surface of both triboelectric layers, respectively. On one side, the inductively coupled plasma (ICP) process was utilized to create nanorods on the surface of the FEP film by reactive ion etching (RIE); ${ }^{27}$ on the other side, we employed the photolithography approach to selectively deposit aluminum patterns of microscale cubic structure onto the flat aluminum foil. The typical scanning electron microscopy (SEM) images of both structures are displayed in Figures $1 \mathrm{~b}$ and 1c. The two triboelectric layers were first brought into contact to produce triboelectric charges through the transfer of electrons from the surface of aluminum to FEP. The surface charges on the dielectric FEP would remain almost unchanged for days, and then the device could be continuously working even when the two layers were not in contact. This is the initial step to have the two surfaces being 

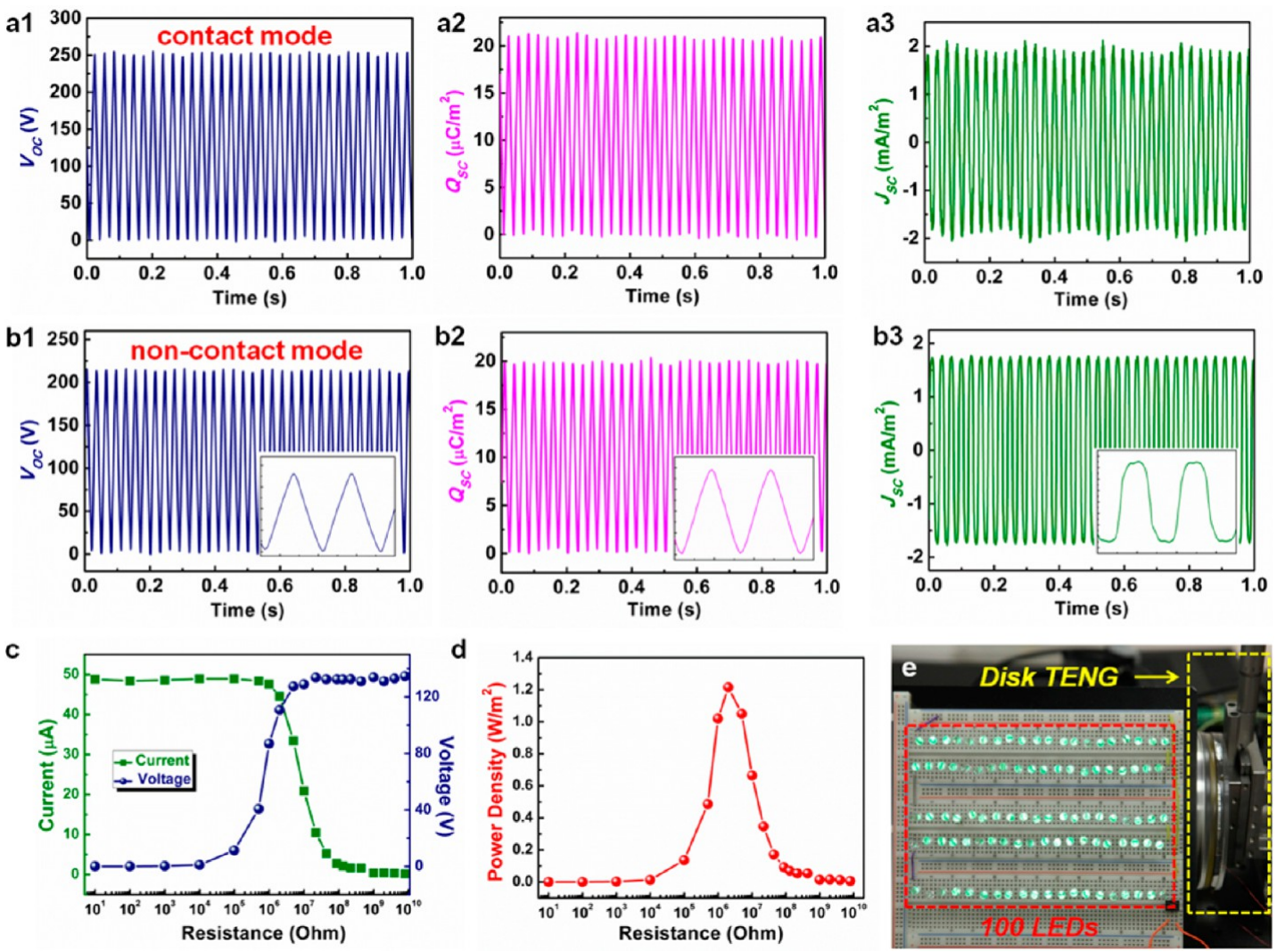

Figure 3. Electrical output characteristics of the FRD-TENG. (a1-a3) The measured $V_{\mathrm{OC}}, Q_{\mathrm{SC}}$, and $J_{\mathrm{SC}}$ of the FRD-TENG at contact mode, respectively. (b1-b3) The measured $V_{\mathrm{OC}}, Q_{\mathrm{SC}}$, and $J_{\mathrm{SC}}$ of the FRD-TENG at noncontact mode, respectively. The insets are the enlarged view showing the detailed shape of each output profile. (c) The measured output voltage and current across an external load with variable resistances. (d) The calculated effective power output of the FRD-TENG with variable resistances. (e) A snapshot from the video showing that the FRD-TENG is able to power up 100 serially connected LED instantaneously and continuously.

electrostatically charged with opposite signs of charges, respectively. The length of time that the electrostatic charges are preserved on the surfaces depends on the nature of the materials and conditions in environment such as humidity. The surface triboelectric charges could even be regenerated by bringing the two surfaces back to contact after a long term of noncontact operation. In the experiment, the rotational part was driven by a rotation motor with tunable rotation speed $(r)$, and the stationary part was fixed on a three-dimensional stage to control the vertical distance $(d)$ between the two parallel triboelectric surfaces.

The working principle of the FRD-TENG is schematically depicted in Figure 2a. For simplicity purposes, all of the schematics in this figure are plotted in a two-dimensional crosssectional view. The energy generation process of the FRDTENG can be divided into two major steps: the initial contact electrification step and the cyclic rotational electrostatic induction step. At first, the FEP segments were brought into contact with one layer of the aluminum foil, which is named as $\mathrm{A} 1$, with the other layer of the aluminum foil as A2. Due to the different triboelectric polarities of the two surfaces, the electrons will be injected from the aluminum foil to the FEP surface, leaving net negative charges on the FEP surface, and net positive charges on the aluminum foil. ${ }^{28-30}$ Then, the two layers were brought apart, leaving a $0.5 \mathrm{~mm}$ thick air media in between. At this right moment, since the vertical separation distance is relatively small compared to the horizontal distance between the mass centers of two adjacent aluminum segments, only few electrons will be driven to flow from A2 to A1 (step I).
As the freestanding segment of FEP is rotating from $\mathrm{A} 1$ to $\mathrm{A} 2$, the electrons will flow from $\mathrm{A} 2$ to $\mathrm{A} 1$ to screen the potential difference generated by the nonmobile negative charges on the FEP surface, producing the first half cycle of the energy conversion process (step II). When the FEP reaches the overlapping position of $\mathrm{A} 2$, the majority of electrons will be driven to A1, leaving most of the positive charges on A2 (step III). In the next step of motion, the segments of FEP will start to move toward the adjacent segment of A1. As a result, the electrons will move back from A1 to A2, leading to an output current with the opposite direction (step IV), until the FEP segments return to the original position, which is the second half of the energy conversion process.

To obtain a quantitative understanding about the working mechanism of the FRD-TENG, numerical calculations on the induced potential difference ${ }^{31,32}$ in different motion steps were carried out using Comsol 4.2a, as displayed in Figure 2b. The three-dimensional illustrations corresponding to this full cycle of motion discussed above are plotted in Figure S1 in the Supporting Information. In the established model, the FEP layer and aluminum layers with the same size and segmental structure were stacked in the vertical direction with an air gap of $0.5 \mathrm{~mm}$, which is the same as the experimental conditions. Initially, the FEP thin film (with an assumed tribo-charge density of $Q_{0}=25 \mu \mathrm{C} / \mathrm{m}^{2}$ ) is fully over $\mathrm{A} 1$, and most of the positive tribo-charges are assumed to distribute on $\mathrm{Al}$ as the minimum achievable charge reference state. As expected, the calculation result shows no potential difference between the two electrodes (step I). In the following step, the negatively 

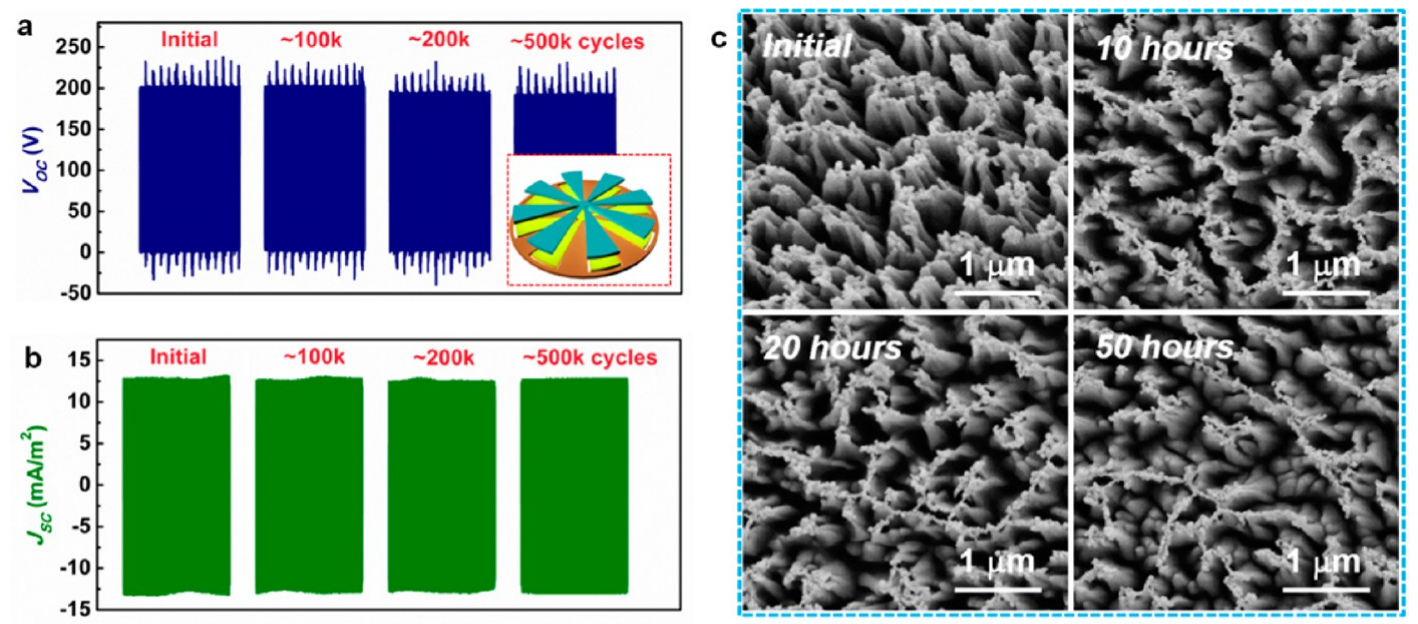

d

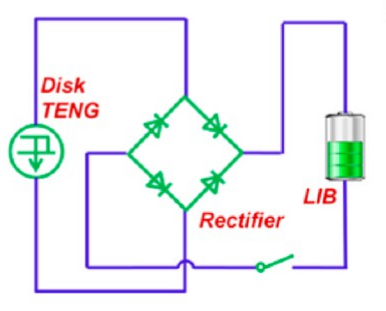

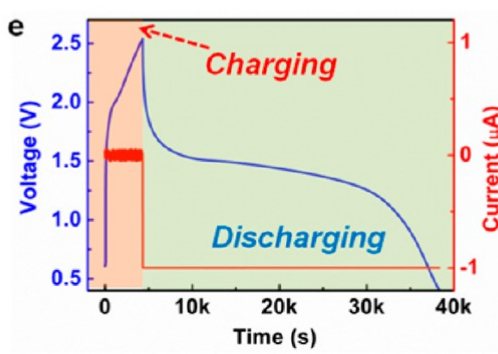

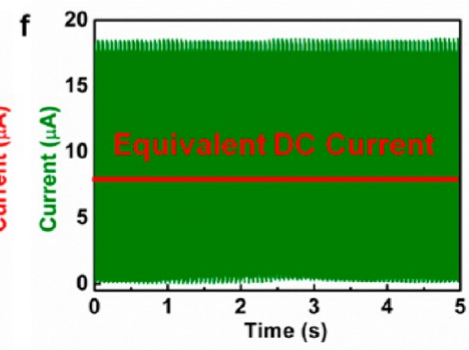

Figure 4. Stability tests of the FRD-TENG and its application for charging a lithium ion battery. (a) The measured results of the $V_{\mathrm{OC}}$ after the FRDTENG had been continuously working for 100000,200000 , and 500000 cycles. The inset shows the schematic structure of the eight-segment device used in this test. (b) The measured results of the $J_{\mathrm{SC}}$ after the FRD-TENG has been continuously working for 100000,200000 , and 500000 cycles. (c) The SEM images of the nanorod structures on the FEP thin film after the FRD-TENG was continuously operated for 10, 20, and 50 h. (d) The schematic circuit diagram for charging a LIB using the FRD-TENG. (e) The measured charging and discharging curve of the LIB as it was first charged by the FRD-TENG at $1000 \mathrm{rpm}$ and consequently discharged at a constant current of $1 \mu \mathrm{A}$. (f) The measured rectified output current of the FRD-TENG at $1000 \mathrm{rpm}$. The red line indicates the equivalent DC charging current calculated from the charging curve of the LIB.

charged FEP surface rotates to the middle position between the two electrodes, resulting in a relatively higher potential difference (step II). As the FEP segments move to the overlapping position over A2 (step III), the calculated induced potential difference reaches its maximum value $(\sim 4 \mathrm{kV})$, and the increase of potential difference provides the driving force for the electrons flowing in the external load. Finally, the FEP layer moves toward the adjacent A1 electrode through the symmetric position with step II with equal potential difference (step IV), until the FEP layer returns to the original position after a full energy conversion cycle.

The typical output characteristics of the FRD-TENG operated at $500 \mathrm{rpm}$ are presented in Figure 3b, and these were compared with the corresponding output performances at contact-mode (Figure 3a). Figure 3a1-3 shows the opencircuit voltage $\left(V_{\mathrm{OC}}\right)$, the transferred charge density $\left(Q_{\mathrm{SC}}\right)$, and the short-circuit current density $\left(J_{S C}\right)$ of the TENG under contact mode $(d=0)$, respectively. Under this condition it can be found that $V_{\mathrm{OC}}=250 \mathrm{~V}, Q_{\mathrm{SC}}=21 \mu \mathrm{C} / \mathrm{m}^{2}$, and $J_{\mathrm{SC}}=2.0 \mathrm{~mA} /$ $\mathrm{m}^{2}$. The corresponding measurement results of the noncontact mode $(d=0.5 \mathrm{~mm})$ are shown in Figure $3 \mathrm{~b} 1-3$. The output performances for the noncontact mode are only slightly lower than those for the contact mode, with $V_{\mathrm{OC}}=220 \mathrm{~V}, Q_{\mathrm{SC}}=20$ $\mu \mathrm{C} / \mathrm{m}^{2}$, and $J_{\mathrm{SC}}=1.8 \mathrm{~mA} / \mathrm{m}^{2}$. These results elucidate the FRDTENG's capability of effectively generating electricity without further friction, indicating that a tight contact during the energy conversion process is no longer necessary. Specifically, it can be found that the output profiles of both the $V_{\mathrm{OC}}$ and the $Q_{\mathrm{SC}}$ are triangular waveform, while the $J_{S C}$ yields square waveform (insets of Figure $3 \mathrm{~b}$ ), which reflects the time-differentiation relationship between the transferred charges and the current. To gain a comprehensive view of the effective output power on the external load, we conducted the measurement of the output voltage and current with variable load resistances, as shown in Figure 3c. Generally, the output voltage increases with the loading resistance while the output current exhibits an opposite trend. Both curves have a quasi-linear region between 0.1 and $100 \mathrm{M} \Omega$, where their variations with the loading resistance become significant. The output power with different resistances could be calculated by simply multiplying the as-measured data of the output current and voltage, as summarized in Figure 3d. Based on this result, it can be found that the maximum output power density of the FRD-TENG is $1.22 \mathrm{~W} / \mathrm{m}^{2}$, at a load resistance of $\sim 2 \mathrm{M} \Omega$. The high output power of the FRDTENG can be utilized for instantaneously and continuously driving 100 LEDs in serial connection, as shown in Figure 3e and Supporting Information Video S1, implying its applications as a self-lighting disk for security monitoring, wind detection, or amusement purposes.

Owing to its unique working principle and the noncontact operation capability, the FRD-TENG has significant advantages compared to the previously demonstrated D-TENG, in the durability of device structure, the stability of its electrical output (especially at very high rotation speed), and the energy conversion efficiency. To demonstrate this superiority of the FRD-TENG, the stability tests were carried out on a device with an eight-segment structure (inset of Figure 4a), at a rotation speed of $3000 \mathrm{rpm}$. At first, all three output parameters 

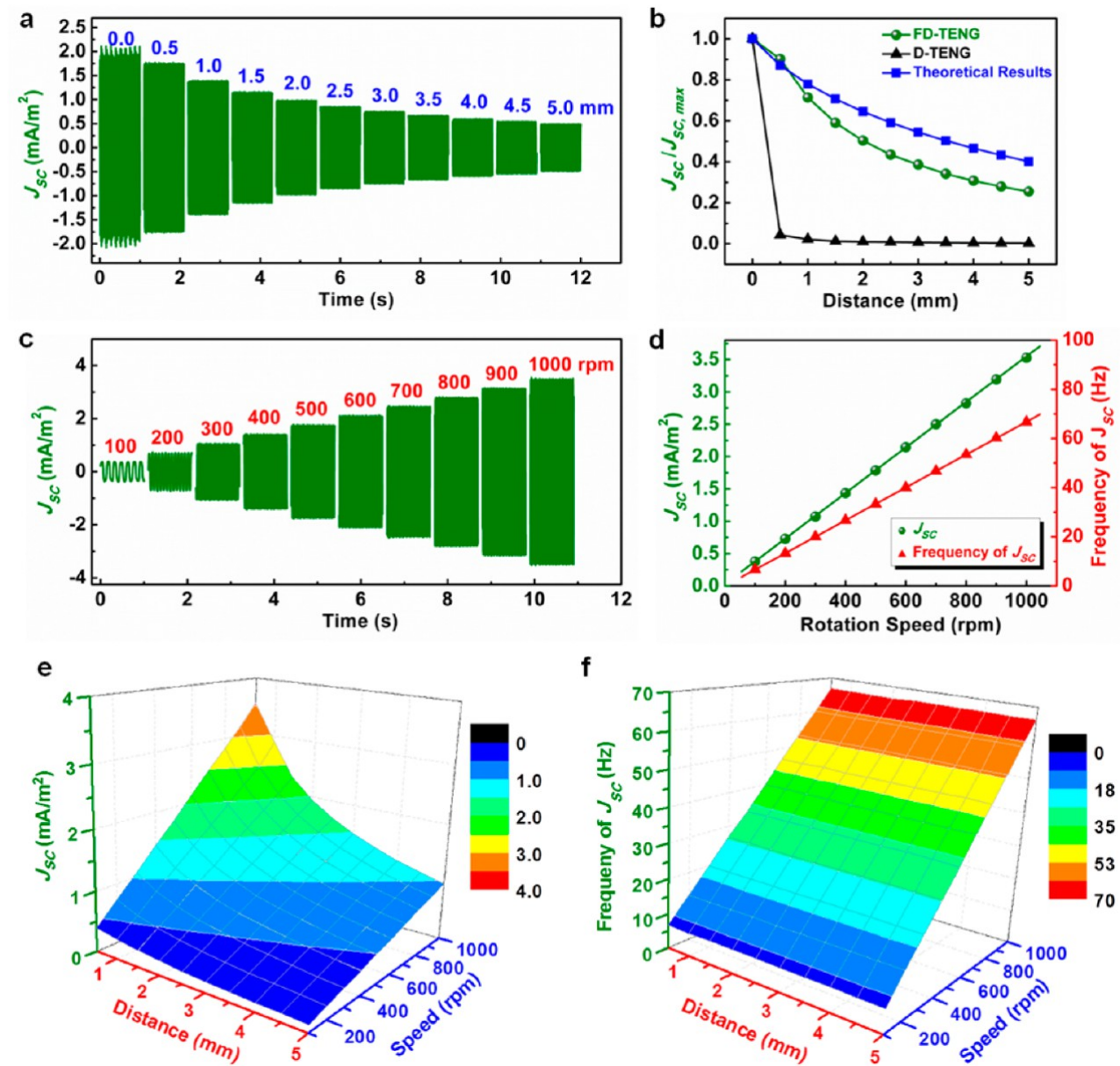

Figure 5. Application of the FRD-TENG as a self-powered mechanical sensor. (a) The measured $J_{\mathrm{SC}}$ with variable vertical distances ranging from 0 to $5 \mathrm{~mm}$. (b) The comparison of the distance dependences on the relative variations of the $J_{\mathrm{SC}}$ between the measured results for the FRD-TENG and the existing D-TENG, as well as the numerical calculation results. (c) The measured $J_{\mathrm{SC}}$ with variable rotation speeds ranging from 100 to $1000 \mathrm{rpm}$. (d) The linear fitting of the measured magnitude and frequency of the $J_{S C}$ with variable rotation speeds. (e) A three-dimensional plot of the magnitude of the $J_{\mathrm{SC}}$ depending on variable vertical displacements and rotation speeds. (f) A three-dimensional plot of the frequency of the $J_{\mathrm{SC}}$ depending on variable vertical displacements and rotation speeds.

of the FRD-TENG were measured before and after a 500000 cycle continuous operation, and it is obvious that they all (Figure 4a, b and Figure S2) display little drops after 500000 cycles of operation. Such supreme stability results from the elimination of the surface abrasion during the noncontact operation. The SEM images of the FEP microstructures were taken after 10,20, and $50 \mathrm{~h}$ of operation in the FRD-TENG, as shown in Figure 4c. It can be observed that the polymer nanorods were rarely affected by the huge numbers of electricity generation cycles, indicating the superhigh durability of the device structure at the microscale.

For most of the energy harvesting techniques, it is needed to store the power scavenged from the environment in an energy storage unit, like a capacitor or a LIB. Because of the unique stability of the FRD-TENG, it can effectively charge a homemade $\mathrm{LIB}\left(\mathrm{LiFePO}_{4}\right.$ as the cathode and $\mathrm{TiO}_{2}$ as the anode) after rectification, which takes much longer charging time than a capacitor. The charging circuit diagram is shown in Figure $4 \mathrm{~d}$. From the measured charging curve in Figure 4e, it can be found that the LIB was successfully charged from $0.7 \mathrm{~V}$ (indicating empty capacity for this battery system) to over 2.5 $\mathrm{V}$ (indicating full capacity) in less than $1.5 \mathrm{~h}$, by the FRDTENG operated under the rotation speed of $1000 \mathrm{rpm}$. The fully charged LIB can provide a constant current of $1 \mu \mathrm{A}$ for around $10 \mathrm{~h}$. We continue to calculate the equivalent constant DC current $\left(I_{\text {equ }}\right)$ indicating that the output current of the TENG has equal effect to a certain constant current when charging an energy storage unit. The $I_{\text {equ }}$ could be calculated from the charging curve by

$$
I_{\text {equ }}=\frac{I_{\text {disch }} t_{\text {disch }}}{t_{\mathrm{ch}}}
$$

Here, $I_{\text {disch }}$ is the constant discharging current, $t_{\text {disch }}$ is the discharging time, and $t_{\mathrm{ch}}$ is the charging time. Based on eq 1 , it can be calculated that $I_{\text {equ }}=7.5 \mu \mathrm{A}$ for this measurement, which is $\sim 41 \%$ of the peak value of the rectified current (Figure $4 \mathrm{f}$ ). The charging curve of the LIB at a relative lower speed (500 $\mathrm{rpm}$ ) was also measured, as plotted in Figure S3, and it was calculated that $I_{\text {equ }}=3.0 \mu \mathrm{A}$ ( $33 \%$ of the peak rectified current), which is lower due to decreasing number of charge transfer cycles in certain time.

In the noncontact operation mode, the vertical distance $(d)$ between the freestanding FEP layer and the fixed aluminum layers is a critical parameter on the output performance. To elucidate its impact, the output performances of the FRDTENG under a series of distances increasing from 0 to $5 \mathrm{~mm}$ were measured at a constant rotation speed of $500 \mathrm{rpm}$. As 
displayed in Figure 5a, the as-measured $J_{\mathrm{SC}}$ shows a decreasing trend from 2.0 to $0.5 \mathrm{~mA} / \mathrm{m}^{2}$, since the electrostatic induction of the negatively charged FEP layer becomes lower. We also employed numerical calculations to gain a theoretical understanding about the impact of the separation distance on the output of the FRD-TENG (Figure S4). As $d$ increases from 0 to $5 \mathrm{~mm}$, the induced potential difference exhibits an obvious descending trend, and the impact on the $Q_{S C}$ and the $J_{S C}$ could then be calculated analytically on the basis of the calculated potential difference, as summarized in Figure $5 \mathrm{~b}$ and Supporting Information Figure S5. For a clear comparison, the relative decay of the $J_{\mathrm{SC}}$ with increasing separation distances was also summarized in Figure $5 b$, together with the calculated results and experimental data for the existing D-TENG. It can be found that the measurement data are in good coherence with the calculated trend, and the FRD-TENG's tolerance of vertical separation is drastically improved compared to the existing D-TENG. For instance, at $d=0.5 \mathrm{~mm}$, the $J_{\mathrm{SC}}$ retains over $90 \%$ of the original value $(d=0)$ for the FRD-TENG, while it drops to only $4.3 \%$ for the D-TENG; as the separation distance goes up to $5 \mathrm{~mm}$, the $J_{\mathrm{SC}}$ still remains $25 \%$ of the original value for the FRD-TENG while it is close to zero $(\sim 0.29 \%)$ for the case of the D-TENG. Similar results were also obtained theoretically and experimentally for the $V_{\mathrm{OC}} / Q_{\mathrm{SC}}$, as summarized in Figure S5. These results demonstrate another unique advantage of the current device structure over the existing design of the D-TENG, ensuring its operation at highstable noncontact mode. For the energy harvesting applications, although the current drops in noncontact mode in comparison to the contact mode D-TENG, the frictional force between two surfaces is minimized so that the consumed mechanical energy is largely reduced as well. Therefore, the FRD-TENG is expected to have much higher energy conversion efficiency than the D-TENG. Moreover, this relationship implies that the FRD-TENG could be employed as a self-powered displacement sensor, which is also visualized through direct powering of LEDs in Video S1. The self-powered displacement sensor has the potential for active detection of the position of a rotating disk without applying an external power source, for unique applications like monitoring the thickness of the braking pad in automobiles.

Besides the separation distance, the rotation speed is another important factor that would have impact on the output performance of the FRD-TENG, especially the current. The $J_{\text {SC }}$ under a series of rotation speeds ranging from 100 to $1000 \mathrm{rpm}$ was measured and displayed in Figure $5 c$, where the vertical distance was kept at $d=0.5 \mathrm{~mm}$. It can be clearly found that the $J_{\mathrm{SC}}$ increases with the rotation speed, since the output current is the time differentiation of the transferred charges across the external load. Since the measured result showing that the $Q_{\mathrm{SC}}$ is independent of the rotation speed (Figure S6), it can be concluded that the $J_{\mathrm{SC}}$ should be linearly related to the rotation speed. This theoretical expectation was verified through the linear fitting of the measured data displayed in Figure 5d. It is also shown in the figure that the frequency of the $J_{S C}$ also exhibits perfect linear relationship to the rotation speed. The above two relationships indicate that the FRD-TENG could be employed as a self-powered rotation speed sensor by analyzing both the magnitude and frequency of the measured $J_{\mathrm{SC}}$. This approach for effectively measuring the rotation speed through either the magnitude or the frequency analysis of the $J_{S C}$ is valid for both the low speed region (as low as $1 \mathrm{rpm}$, Figure S7) and the high speed region (up to $2500 \mathrm{rpm}$, Figure S8).
From the above discussion, the rotation speed and the vertical separation distance are two input parameters influencing the output current signal, which can be characterized by the magnitude and the frequency. To illustrate this twofold relationship and show a comprehensive working characteristic of the FRD-TENG as a self-powered sensor, we carried out two sets of experiments to give three-dimensional plots of the twofold dependence of the magnitude (Figure 5e) and frequency (Figure 5f) on the vertical displacement and the rotation speed, respectively. As expected, the magnitude was affected by both the vertical displacement and the rotation speed, while the frequency was only dependent on the rotation speed. From these two graphs, the information about two important parameters of a mechanical motion could be solely determined by the measured profiles of the $J_{\mathrm{SC}}$. Based on this principle, the FRD-TENG could be used as a self-powered mechanical sensor in automobiles to detect both the radial and axial movement of a braking pad at the same time. This twoparameter monitoring approach provides us deeper insights on the application of nanogenerator in self-powered system and exhibits unique advantages than the existing single-parameter active sensors. ${ }^{33,34}$

\section{SUMMARY}

In summary, we have introduced a novel disk structure that is capable of effectively harvesting the rotational mechanical energy at the noncontact mode. The working principle of the noncontact free-rotating disk triboelectric nanogenerator has been elucidated via conjunction of schematic illustrations, numerical calculations, as well as experimental examinations. Compared with the existing disk triboelectric nanogenerator, the FRD-TENG in this work has displayed numerous advantages including much higher stability and durability, and much smaller deterioration in performance at the noncontact mode. The high stability and large output density ensures its usage in fast and effective charging of a lithium ion battery, showing its potential to scavenge the energy from the braking system to charge the battery in the automobile. Finally, based on the relationship between the output characteristics and the external mechanical triggering, the FRD-TENG could be employed as a self-powered sensor for reliable measurement of the vertical displacement and the rotation speed simultaneously.

\section{EXPERIMENTAL SECTION}

Fabrication of the Nanorod Structures on the Surface of the FEP Thin Film. A $50 \mu \mathrm{m}$ thick FEP thin film was cleaned with menthol, isopropyl alcohol, and deionized water, consecutively, and then blown dry with nitrogen gas. Then, the surface of the FEP thin film was deposited with gold thin film (10 nm in thickness) by sputtering (Unifilm Sputter) as the mask for the following etching to create the nanorod structures using ICP. Specifically, $\mathrm{Ar}, \mathrm{O}_{2}$, and $\mathrm{CF}_{4}$ gases were introduced into the ICP chamber with the flow rate of 15.0, 10.0 , and $30.0 \mathrm{sccm}$, respectively. One power source of $400 \mathrm{~W}$ was used to generate a high density of plasma and the other power of 100 $\mathrm{W}$ was used to accelerate the plasma ions. The FEP thin film was etched for 1-5 min with the above parameters.

Fabrication of the Micropatterned Structures on the Surface of the Aluminum Foil. A $50 \mu \mathrm{m}$ thick aluminum foil was patterned with a typical photolithography process: defining the photoresist to the array of square windows, depositing a layer of aluminum on top, and finally lift-off, leaving the patterned $\mathrm{Al}$ cubes on the foil.

Fabrication of the Noncontact Free-Rotating Disk Triboelectric Nanogenerator. First, two $0.125^{\prime \prime}$ thick acrylic sheets were processed by laser cutting (PLS6.75, Universal Laser Systems) to form 
the two cyclostyles as supporting substrates. The tailored aluminum foils and FEP thin film were securely attached on the acrylic loading templates to make the surfaces of the aluminum foils and the FEP thin film parallel to each other.

Electrical Measurement of the Noncontact Free-Rotating Disk Triboelectric Nanogenerator. In the electrical output measurement, the rotational part of the FRD-TENG was bonded onto a spinning motor (BX460AM-A, Oriental Motor), and the stationary part was secured on a stationary XYZ linear translation stage (462-XYZ-M, Newport Incorporation), with both centers of the disks in coincidence with the spinning axis. The FEP segments were driven to rotate around the axis of the motor with variable rotating speeds, and vertical distance between both parts was accurately controlled by the linear translation stage. The open-circuit voltage and transferred charge density were measured by using a Keithley 6514 System electrometer, and the short-circuit current was measured by using an SR570 low noise current amplifier (Stanford Research System).

\section{ASSOCIATED CONTENT}

\section{(S Supporting Information}

Additional schematic figures, calculations on the distance dependence of potential difference, and more characterization data of the self-powered displacement/speed sensor. This material is available free of charge via the Internet at http:// pubs.acs.org.

\section{AUTHOR INFORMATION}

\section{Corresponding Author}

*E-mail: zlwang@gatech.edu.

\section{Author Contributions}

${ }^{\S}$ L.L., S.W., and S.N. contributed equally to this work.

\section{Notes}

The authors declare no competing financial interest.

\section{ACKNOWLEDGMENTS}

Research was supported by MANA, NIMS, Japan, the "thousands talents" program for pioneer researcher and his innovation team, China, and Beijing City Committee of science and technology project (Z131100006013004).

\section{REFERENCES}

(1) Lele, S. M. Sustainable Development - a Critical Review. World Dev. 1991, 19, 607-621.

(2) Meyar-Naimi, H.; Vaez-Zadeh, S. Sustainable Development Based Energy Policy Making Frameworks, a Critical Review. Energy Policy 2012, 43, 351-361.

(3) Dresselhaus, M. S.; Thomas, I. L. Alternative Energy Technologies. Nature 2001, 414, 332-337.

(4) Gratzel, M. Photoelectrochemical Cells. Nature 2001, 414, 338344.

(5) Oregan, B.; Gratzel, M. A Low-Cost, High-Efficiency Solar-Cell Based on Dye-Sensitized Colloidal $\mathrm{TiO}_{2}$ Films. Nature 1991, 353, 737-740.

(6) Mitcheson, P. D.; Yeatman, E. M.; Rao, G. K.; Holmes, A. S.; Green, T. C. Energy Harvesting from Human and Machine Motion for Wireless Electronic Devices. Proc. IEEE 2008, 96, 1457-1486.

(7) Wang, Z. L.; Song, J. H. Piezoelectric Nanogenerators Based on Zinc Oxide Nanowire Arrays. Science 2006, 312, 242-246.

(8) Chang, C. E.; Tran, V. H.; Wang, J. B.; Fuh, Y. K.; Lin, L. W. Direct-Write Piezoelectric Polymeric Nanogenerator with High Energy Conversion Efficiency. Nano Lett. 2010, 10, 726-731.

(9) Chen, X.; Xu, S. Y.; Yao, N.; Shi, Y. 1.6 V Nanogenerator for Mechanical Energy Harvesting Using PZT Nanofibers. Nano Lett. 2010, 10, 2133-2137.

(10) Mitcheson, P. D.; Miao, P.; Stark, B. H.; Yeatman, E. M.; Holmes, A. S.; Green, T. C. MEMS Electrostatic Micropower
Generator for Low Frequency Operation. Sens. Actuators, A 2004, $115,523-529$.

(11) Naruse, Y.; Matsubara, N.; Mabuchi, K.; Izumi, M.; Suzuki, S. Electrostatic Micro Power Generation from Low-Frequency Vibration Such as Human Motion. J. Micromech. Microeng. 2009, 19, 094002.

(12) El-hami, M.; Glynne-Jones, P.; White, N. M.; Hill, M.; Beeby, S.; James, E.; Brown, A. D.; Ross, J. N. Design and Fabrication of a New Vibration-Based Electromechanical Power Generator. Sens. Actuators, A 2001, 92, 335-342.

(13) Williams, C. B.; Shearwood, C.; Harradine, M. A.; Mellor, P. H.; Birch, T. S.; Yates, R. B. Development of an Electromagnetic MicroGenerator. IEE Proc. Circuits Devices Syst. 2001, 148, 337-342.

(14) Beeby, S. P.; Torah, R. N.; Tudor, M. J.; Glynne-Jones, P.; O’Donnell, T.; Saha, C. R.; Roy, S. A Micro Electromagnetic Generator for Vibration Energy Harvesting. J. Micromech. Microeng. 2007, 17, 1257-1265.

(15) Wang, Z. L. Triboelectric Nanogenerators as New Energy Technology for Self-Powered Systems and as Active Mechanical and Chemical Sensors. ACS Nano 2013, 7, 9533-9557.

(16) Fan, F. R.; Tian, Z. Q.; Wang, Z. L. Flexible Triboelectric Generator. Nano Energy 2012, 1, 328-334.

(17) Fan, F. R.; Lin, L.; Zhu, G.; Wu, W. Z.; Zhang, R.; Wang, Z. L. Transparent Triboelectric Nanogenerators and Self-Powered Pressure Sensors Based on Micropatterned Plastic Films. Nano Lett. 2012, 12, 3109-3114.

(18) Yang, Y.; Zhang, H. L.; Chen, J.; Jing, Q. S.; Zhou, Y. S.; Wen, X. N.; Wang, Z. L. Single-Electrode-Based Sliding Triboelectric Nanogenerator for Self-Powered Displacement Vector Sensor System. ACS Nano 2013, 7, 7342-7351.

(19) Zhu, G.; Pan, C. F.; Guo, W. X.; Chen, C. Y.; Zhou, Y. S.; Yu, R. M.; Wang, Z. L. Triboelectric-Generator-Driven Pulse Electrodeposition for Micropatterning. Nano Lett. 2012, 12, 4960-4965.

(20) Wang, S. H.; Lin, L.; Wang, Z. L. Nanoscale TriboelectricEffect-Enabled Energy Conversion for Sustainably Powering Portable Electronics. Nano Lett. 2012, 12, 6339-6346.

(21) Wang, S. H.; Lin, L.; Xie, Y. N.; Jing, Q. S.; Niu, S. M.; Wang, Z. L. Sliding-Triboelectric Nanogenerators Based on In-Plane ChargeSeparation Mechanism. Nano Lett. 2013, 13, 2226-2233.

(22) Lin, L.; Wang, S. H.; Xie, Y. N.; Jing, Q. S.; Niu, S. M.; Hu, Y. F.; Wang, Z. L. Segmentally Structured Disk Triboelectric Nanogenerator for Harvesting Rotational Mechanical Energy. Nano Lett. 2013, 13, 2916-2923.

(23) Jacobs, H. O.; Whitesides, G. M. Submicrometer Patterning of Charge in Thin-Film Electrets. Science 2001, 291, 1763-1766.

(24) Wang, Z. L. Nanosize Machines Need Still Tinier Power Plants. Sci. Am. 2008, 298, 82-87.

(25) Lin, L.; Jing, Q. S.; Zhang, Y.; Hu, Y. F.; Wang, S. H.; Bando, Y.; Han, R. P. S.; Wang, Z. L. An Elastic-Spring-Substrated Nanogenerator as an Active Sensor for Self-Powered Balance. Energy Environ. Sci. 2013, 6, 1164-1169.

(26) Lin, Z. H.; Zhu, G.; Zhou, Y. S.; Yang, Y.; Bai, P.; Chen, J.; Wang, Z. L. A Self-Powered Triboelectric Nanosensor for Mercury Ion Detection. Angew. Chem., Int. Ed. 2013, 52, 5065-5069.

(27) Fang, H.; Wu, W. Z.; Song, J. H.; Wang, Z. L. Controlled Growth of Aligned Polymer Nanowires. J. Phys. Chem. C 2009, 113, 16571-16574.

(28) Diaz, A. F.; Felix-Navarro, R. M. A Semi-Quantitative TriboElectric Series for Polymeric Materials: the Influence of Chemical Structure and Properties. J. Electrost. 2004, 62, 277-290.

(29) McCarty, L. S.; Whitesides, G. M. Electrostatic Charging Due to Separation of Ions at Interfaces: Contact Electrification of Ionic Electrets. Angew. Chem., Int. Ed. 2008, 47, 2188-2207.

(30) Wiles, J. A.; Grzybowski, B. A.; Winkleman, A.; Whitesides, G. M. A Tool for Studying Contact Electrification in Systems Comprising Metals and Insulating Polymers. Anal. Chem. 2003, 75, 4859-4867.

(31) Niu, S. M.; Liu, Y.; Wang, S. H.; Lin, L.; Zhou, Y. S.; Hu, Y. F.; Wang, Z. L. Theory of Sliding-Mode Triboelectric Nanogenerators. Adv. Mater. 2013, 25, 6184-6193. 
(32) Wang, S. H.; Xie, Y. N.; Niu, S. M.; Lin, L.; Wang, Z. L. Freestanding-Triboelectric-Layer Based Nanogenerators for Harvesting Energy from a Moving Object or Human Motion in Contact and Non-Contact Modes. Adv. Mater. 2013, DOI: 10.1002/ adma.201305303.

(33) Lin, L.; Xie, Y. N.; Wang, S. H.; Wu, W. Z.; Niu, S. M.; Wen, X. N.; Wang, Z. L. Triboelectric Active Sensor Array for Self-Powered Static and Dynamic Pressure Detection and Tactile Imaging. ACS Nano 2013, 7, 8266-8274.

(34) Lin, L.; Hu, Y. F.; Xu, C.; Zhang, Y.; Zhang, R.; Wen, X. N.; Wang, Z. L. Transparent Flexible Nanogenerator as Self-Powered Sensor for Transportation Monitoring. Nano Energy 2013, 2, 75-81. 


\title{
Supporting Information
}

\section{Non-contact free-rotating disk triboelectric nanogenerator as a sustainable energy harvester and self-powered mechanical sensor}

Long Lin ${ }^{\dagger, \S}$, Sihong Wang ${ }^{\dagger, \S}$, Simiao $\mathrm{Niu}^{\dagger, \S}$, Chang Liu ${ }^{\dagger}$, Yannan Xie ${ }^{\dagger}$, Zhong Lin Wang ${ }^{*, \dagger, \sharp}$

\author{
*Email: zlwang@gatech.edu \\ ${ }^{\S}$ These authors contributed equally to this work. \\ ${ }^{\dagger}$ School of Materials Science and Engineering, Georgia Institute of Technology, Atlanta, Georgia \\ 30332-0245, United States \\ ${ }^{\ddagger}$ Beijing Institute of Nanoenergy and Nanosystems, Chinese Academy of Sciences, Beijing, \\ 100083, China
}



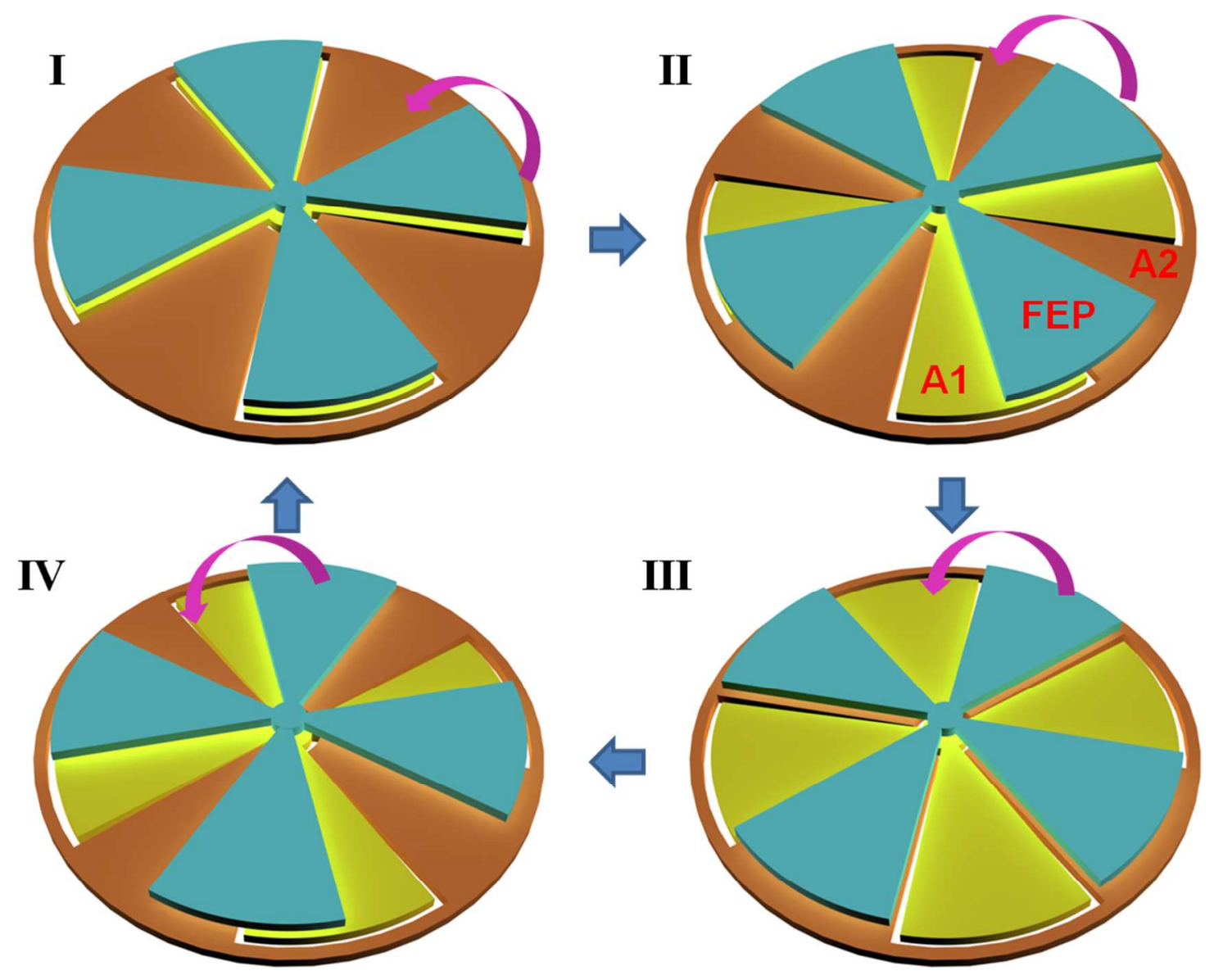

Figure S1. Three-dimensional schematic illustrations showing the relative motion of the FEP segments on the fixed aluminum electrodes in a full electricity generation cycle. 


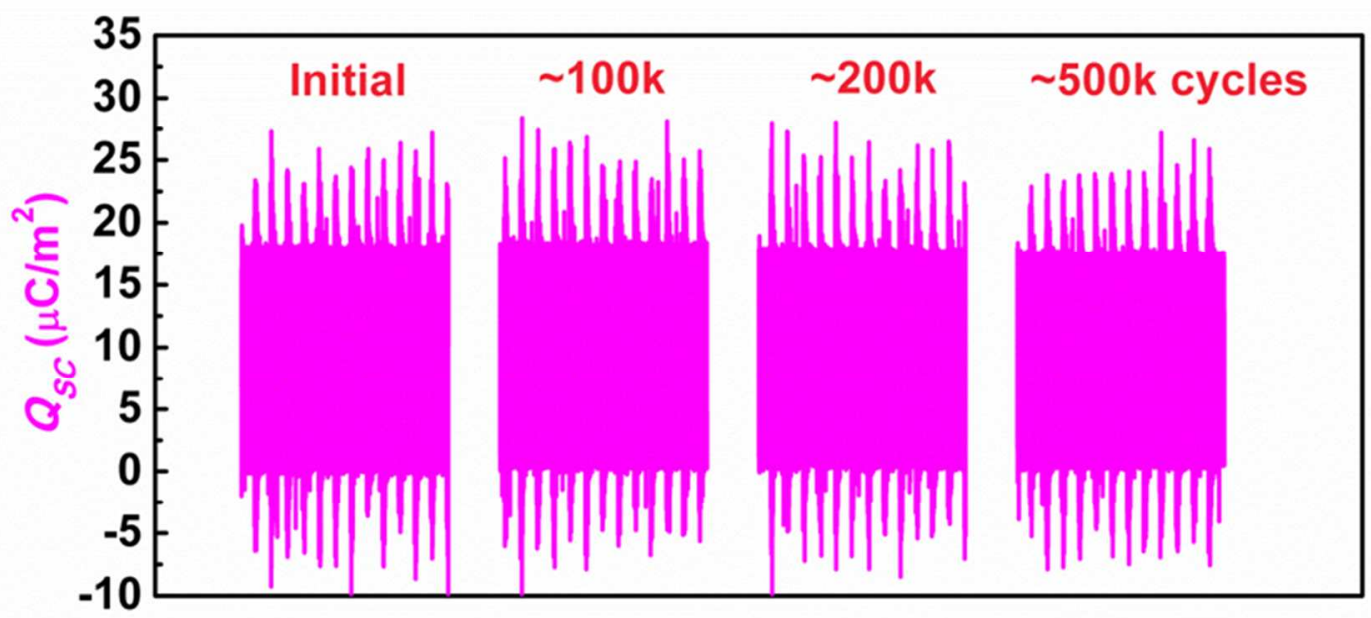

Figure S2. The measured results of the $Q_{S C}$ after continuous working of the FRD-TENG for $100,000,200,000$, and 500,000 cycles, respectively. 

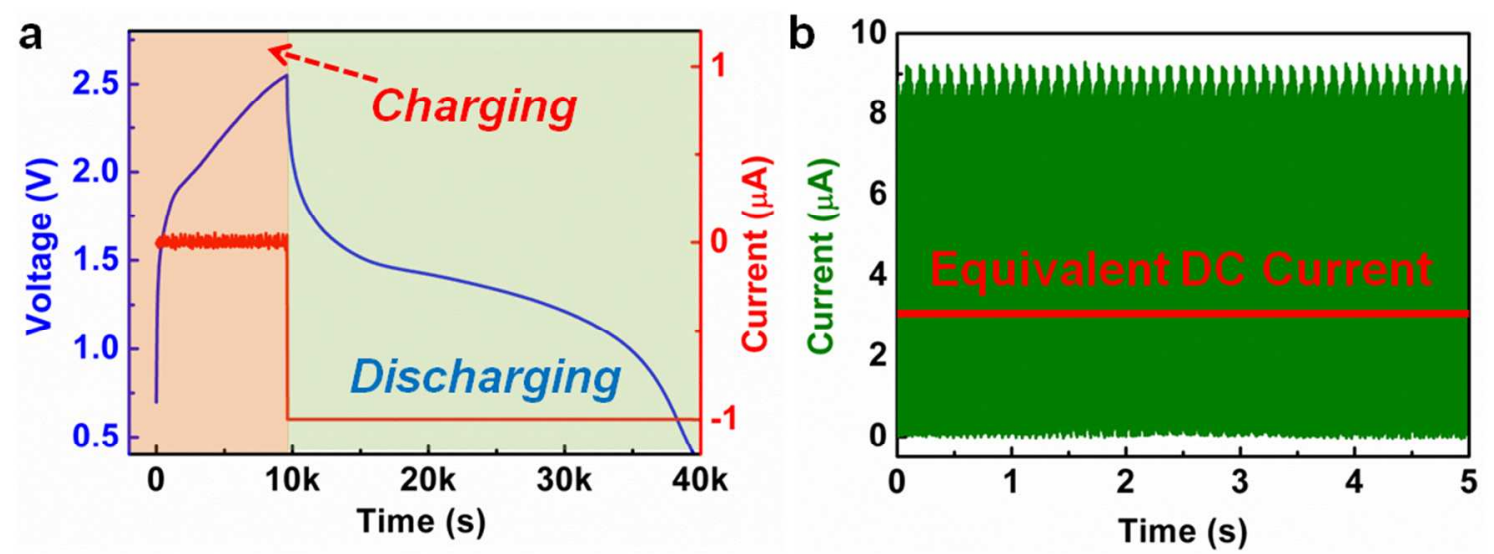

Figure S3. (a) The measured charging and discharging curve of the LIB as it was first charged by the FRD-TENG at $500 \mathrm{rpm}$ and consequently discharged at a constant current of $1 \mu \mathrm{A}$. (f) The measured rectified output current of the FD-TENG at $500 \mathrm{rpm}$. The red line indicates the calculated equivalent DC charging current from the charging curve of the LIB. 


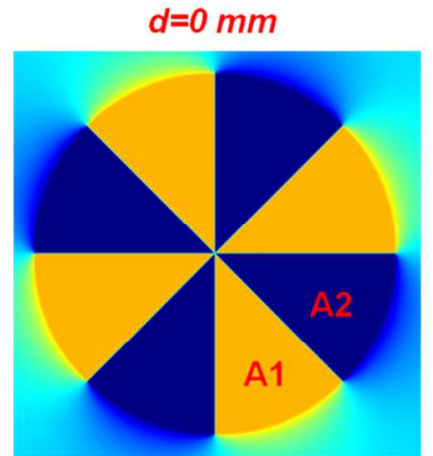

$d=3 \mathrm{~mm}$

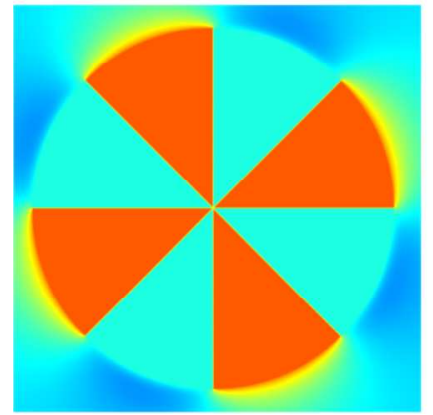

$d=1 \mathrm{~mm}$

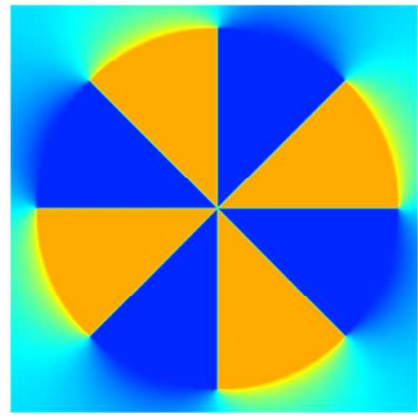

$d=4 \mathrm{~mm}$

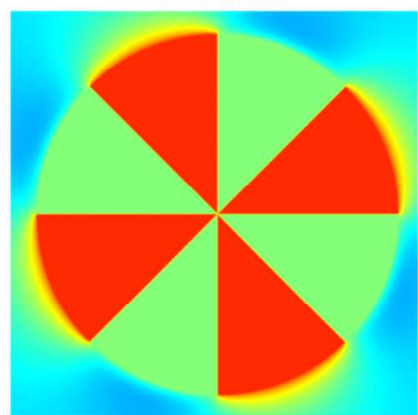

$d=2 \mathrm{~mm}$

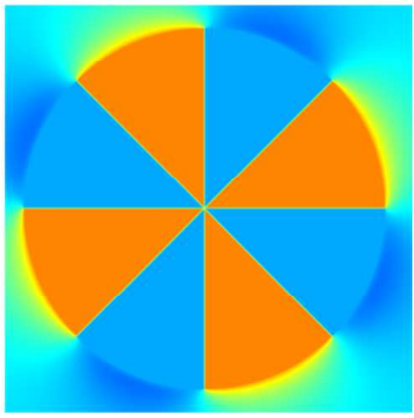

$d=5 \mathrm{~mm}$

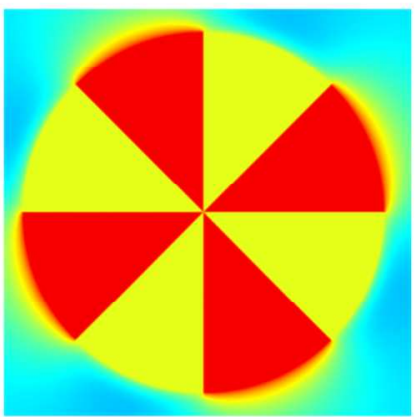

Potential (V)

4000

3000

2000

1000

0

$-1000$

$-2000$

Figure S4. The numerical calculation results showing the induced potential differences between the two aluminum electrodes with variable vertical separation distances ranging from 0 to $5 \mathrm{~mm}$. 

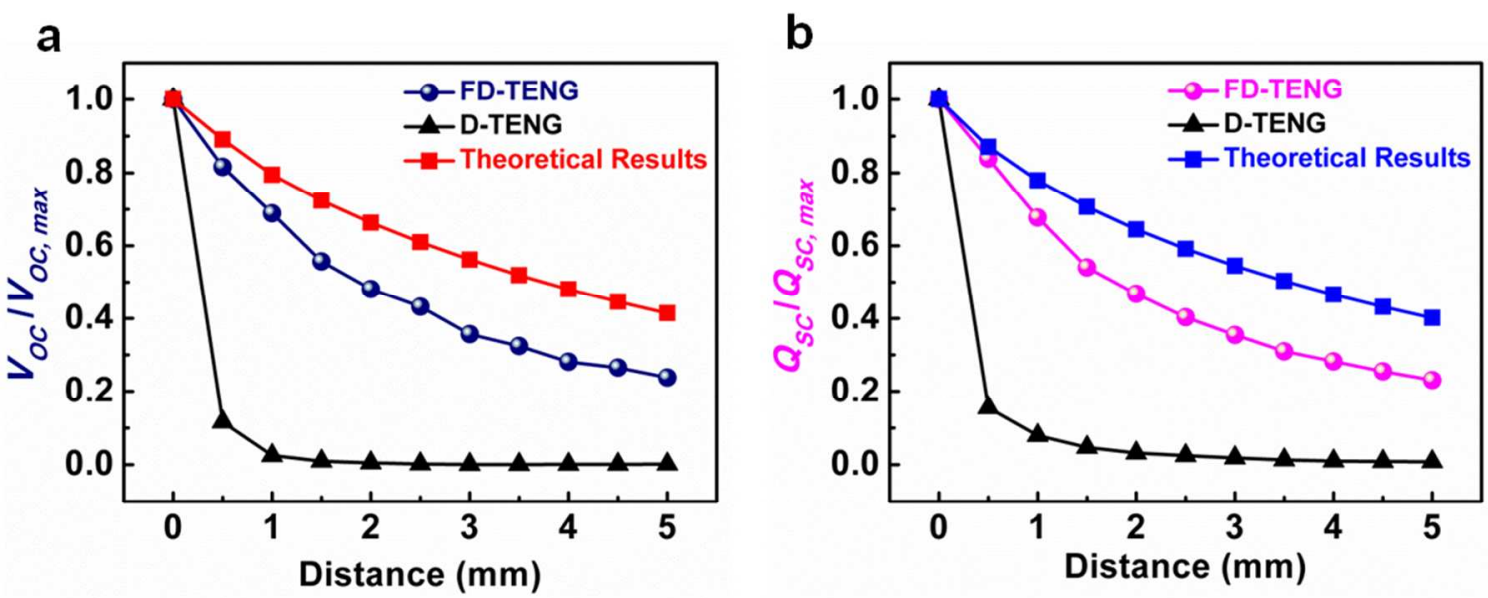

Figure S5. The comparison of the distance dependences on the relative variations of the $V_{O C}(\mathrm{a})$ and the $Q_{S C}(\mathrm{~b})$ between the measured results for the FRD-TENG and the conventional D-TENG, as well as the numerical calculation results. 

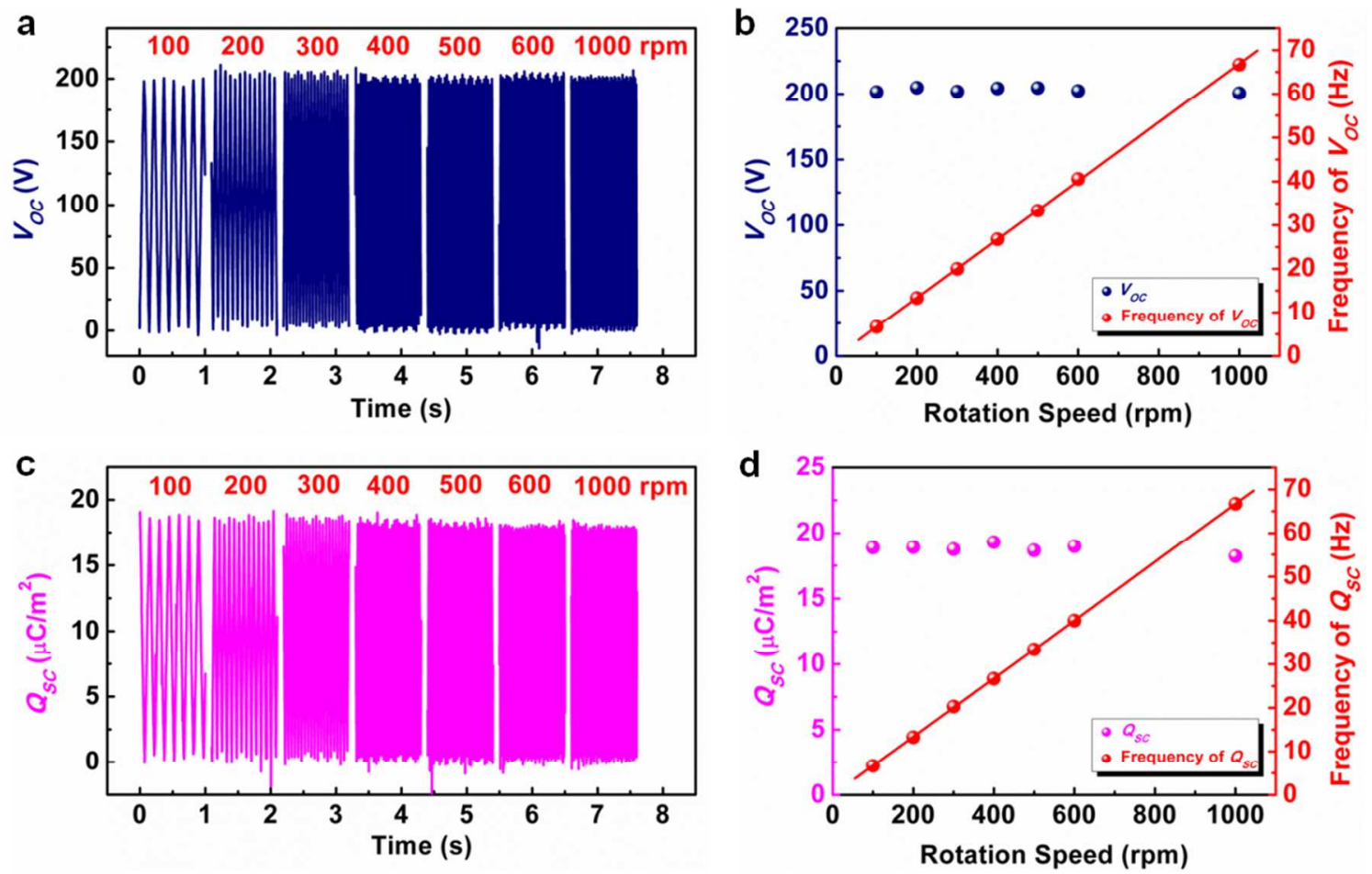

Figure S6. (a) The measured $V_{O C}$ with variable rotation speeds ranging from 100 to $1000 \mathrm{rpm}$. (b) The summation of the measured magnitude and the linear fitting of the measured frequency of the $V_{O C}$ with variable rotation speeds. (c) The measured $Q_{S C}$ with variable rotation speeds ranging from 100 to $1000 \mathrm{rpm}$. (d) The summation of the measured magnitude and the linear fitting of the measured frequency of the $Q_{S C}$ with variable rotation speeds. 

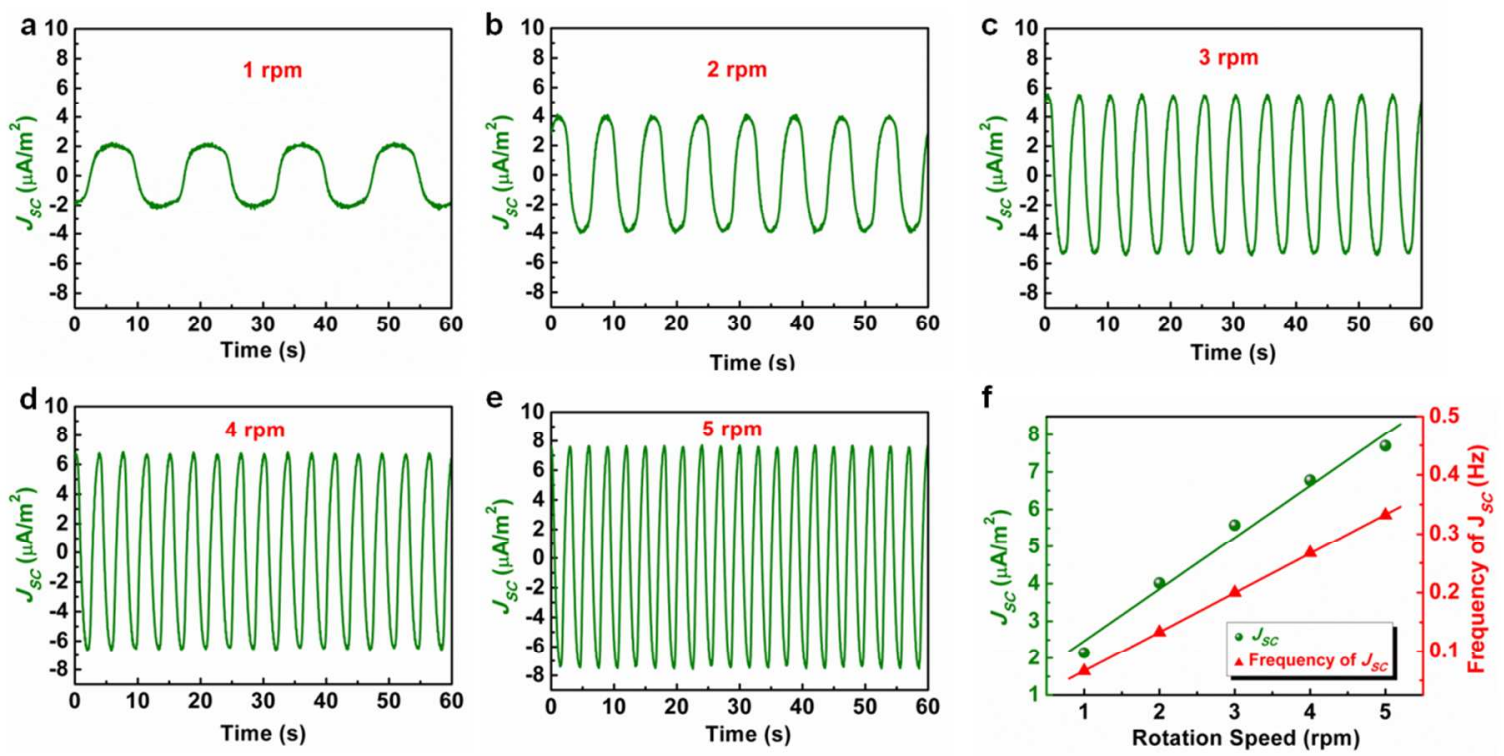

Figure S7. The FRD-TENG as a self-powered mechanical sensor for measuring the rotation speed at the low speed region. (a-e) The measured $J_{S C}$ with variable rotation speeds at 1, 2, 3, 4, and $5 \mathrm{rpm}$, respectively. (f) The linear fitting of the measured magnitude and frequency of the $J_{S C}$ with variable rotation speeds at the low speed region. 

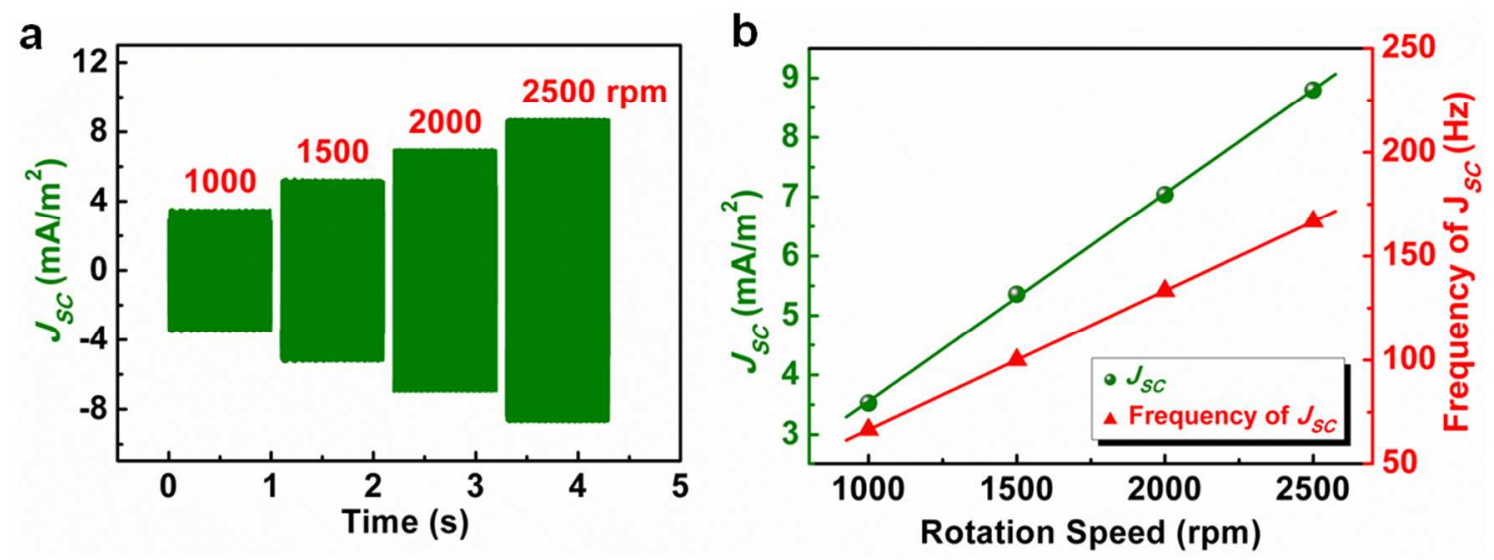

Figure S8. The FRD-TENG as a self-powered mechanical sensor for measuring the rotation speed at the high speed region. (a-e) The measured $J_{S C}$ with variable rotation speeds at 1000 , 1500, 2000, and $2500 \mathrm{rpm}$, respectively. (f) The linear fitting of the measured magnitude and frequency of the $J_{S C}$ with variable rotation speeds at the high speed region.

Video S1. This video shows the continuous powering of 100 serially-connected LEDs by the rectified high output of the FRD-TENG. It also shows in the video that the FRD-TENG could be employed as a self-powered displacement sensor visualized by the light intensity of the LED bulbs when changing the vertical distances. 